26 years, range 19-58 years, 53 male) in a randomised crossover design. Validated questionnaires and visual analogue scales (VAS) were used for anxiety and nausea assessment. We monitored validated measures of autonomic and electrogastrographic activity at baseline and continuously thereafter. Subjects were stratified into quartiles based on nausea VAS scores with the upper and lower quartiles considered to be nausea sensitive and resistant respectively. Of these, 28 subjects of the 50 (mean age 25 years, range $20-49$ years, 16 males, 11 nausea resistant) were exposed to the motion video during fMRI.

Results All subjects completed the studies without vomiting. The motion video induced nausea in 57/98 subjects (57\%) associated with elevation of median nausea VAS scores (2.0 (IRQ 1-3) vs. 1.0 (IRQ $1-1), \mathrm{p}=0.003)$. Nausea sensitive subjects had lower normogastria:tachygastria $(\mathrm{p}=0.048)$, increased sympathetic $(\mathrm{p}=$ $0.002)$ and decreased parasympathetic tone $(p=0.03)$ during the motion video in comparison to the control video. The motion video resulted in heightened neuronal activity in the left and right cerebrum, temporal lobe, middle temporal gyrus and occipital lobe $(\mathrm{p}<0.004)$. Compared to nausea resistant subjects, the nausea sensitive group showed a paucity of activity in the left cerebrum, limbic areas and anterior cingulate cortex $(p<0.001)$.

Conclusion This study provides evidence to validate the motion video as a VIMS stimulus. Additionally, it demonstrates the cortical and psychophysiological changes induced by VIMS. These changes are as a result of the activation of a broad central network, reflecting the multi-dimensional nature of nausea. Sensitivity to VIMS may therefore be as a consequence of failure of, rather than excessive, activation of cortical areas concerned with the interoceptive and affective aspects of nausea.

Disclosure of Interest None Declared.

\section{OC-065 FUNCTIONAL CORTICAL SWALLOWING ACTIVITY AND NEUROTRANSMITTERS CONCENTRATIONS ARE ALTERED FOLLOWING NEUROSTIMULATION OF PHARYNGEAL MOTOR CORTEX: AN FMRI AND RESONANCE SPECTROSCOPY (MRS) STUDY}

${ }^{1} E$ Michou*, ${ }^{1} S$ Mistry, ${ }^{2} R$ Vidyasagar, ${ }^{3} D$ Downey, ${ }^{2} S$ Williams, 'S Hamdy. ${ }^{1}$ Gastrointestinal Sciences, University of Manchester, Manchester, UK; ${ }^{2}$ Centre for Imaging Sciences, University of Manchester, Manchester, UK; ${ }^{3}$ Institute of Brain, Behaviour and Mental Health, University of Manchester, Manchester, UK

\subsection{6/gutjnl-2014-307263.65}

Introduction Combined pharyngeal electrical and transcranialmagnetic-stimulation or paired associative stimulation (PAS) is shown to induce beneficial neurophysiological and behavioural effects on swallowing in health and dysphagic stroke patients (Michou et al., Gastroenterology 2012). Here, we investigate brain changes during swallowing following application of PAS, using fMRI and MR spectroscopy to measure neural activity and GABA concentrations in the motor cortices.

Methods Healthy adults $(\mathrm{n}=11,38 \pm 9$ yoa) were randomised to receive real and sham PAS on 2 separate visits. Event-related fMRI was performed to assess changes in brain activations in response to water and saliva swallowing and during rest. Data were analysed (SPM8), applying $\mathrm{p}<0.001$ uncorrected thresholds with contrasts of 'water swallowing-rest' and 'saliva swallowing-rest'. MRS data were acquired before and after the fMRI on both visits and GABA concentrations were measured (AMARES, jMRUI).

Results Following real PAS, group analyses of 'water swallowing-rest' and 'saliva swallowing-rest' showed increased activation
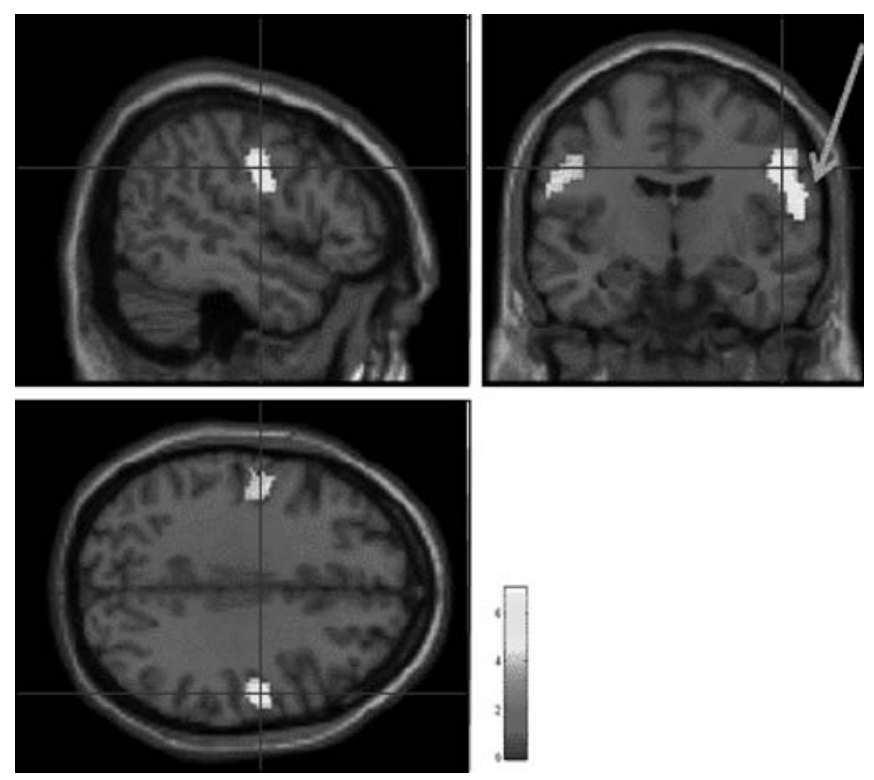

Abstract OC-065 Figure 1

in motor and premotor areas bilaterally. Moreover, real PAS increased activations prominently in premotor areas contralateral to PAS (Figure1 group mean brain activations following real PAS). Following real PAS, GABA concentrations in motor cortex decreased significantly both ipsilateral $(P=0.008)$ and contralateral $(\mathrm{P}=0.013)$ to PAS.

Conclusion Targeted neurostimulation applied to the human pharyngeal motor cortex induces local and remote changes in both primary and non-primary areas for water and saliva tasks. Moreover, stimulation leads to reduction of the inhibitory neurotransmitter GABA, when associated with swallowing. These findings allow us to understand the mechanisms underlying the beneficial effects of neurostimulation in modulating the brain swallowing network.

Disclosure of Interest None Declared.

\section{OC-066 INFLUENCE OF EXTRAVERSION ON BRAIN ACTIVITY AT BASELINE, PAIN ANTICIPATION AND VISCERAL PAIN PROCESSING}

${ }^{1} \mathrm{JK}$ Ruffle*, ${ }^{1} \mathrm{~S} J$ Coen, ${ }^{1} \mathrm{AD}$ Farmer, ${ }^{2} \mathrm{M}$ Kano, ${ }^{3} \mathrm{~V}$ Giampietro, ${ }^{3} \mathrm{~S}$ Williams, ${ }^{1} \mathrm{Q}$ Aziz. ${ }^{1}$ Wingate Institute of Neurogastroenterology, Centre for Gastroenterology, Blizard Institute, Barts and the London School of Medicine and Dentistry, Queen Mary University of London, UK, London, UK; ${ }^{2}$ Behavioural Medicine, Tohoku University, Sendai, Japan; ${ }^{3}$ Department of Neuroimaging, Institute of Psychiatry, Kings College London, London, UK

\subsection{6/gutjnl-2014-307263.66}

Introduction Eysenck proposed 'trait theory' of personality, where the dimensions extraversion (degree of optimism and sociability) and neuroticism (degree of anxiety and fear) encompass numerous individual qualities. Whilst the influence of neuroticism on the brain processing of pain is well studied, the role of extraversion in pain processing remains to be investigated and thus this was the aim of our study using functional magnetic resonance imaging (fMRI).

Methods 33 healthy volunteers participated in the study, all of whom consented in writing (17 male; mean age 29 , range $20-53$, all right handed). Extraversion was measured using the Eysenck Personality Questionnaire. fMRI data was acquired using a 3T GE MRI scanner during rest, anticipation of pain, and painful distal 
oesophageal balloon distention. During fMRI, 480 T2* weighted images per slice $(40 \times 3 \mathrm{~mm}$ slices, 0.3 interslice gap, TE $30 \mathrm{~ms}$, TR $2500 \mathrm{~ms}$, flip angle $80^{\circ}$, matrix size 642, sum of images per scan $=19,200)$ were captured to illustrate blood oxygen level dependent (BOLD) contrast during the different experimental events. The effects of extraversion on fMRI response during these events were subsequently determined using ANOVA brain activation mapping analyses within XBAM, a statistical package of image processing and statistical inference.

Results There was a diversity of extraversion scores (range 622), which did not influence pain threshold or rating. High extraversion was associated with significantly greater activity in the left cuneus (Brodmann Area (BA) 18) during rest ( $\mathrm{p}<$ $0.001)$ and the right insula (BA13) during both anticipation ( $\mathrm{p}<$ $0.0002)$ and pain $(p<0.0008)$. Low extraversion was associated with significantly greater brain activity in numerous regions during pain anticipation, including the bilateral precuneus (BA31), bilateral lingual gyrus (BA18) and the right inferior temporal gyrus $(\mathrm{p}<0.0001)$.

Conclusion Our results suggest that the brain processing of pain is influenced by the personality dimension of extraversion and therefore like other personality dimensions such as neuroticism, extraversion should be controlled for in brain imaging studies of pain in health and disease.

Disclosure of Interest None Declared.

\section{OC-067 ENHANCED PERCEPTION OF PROXIMAL GASTRO-OESOPHAGEAL REFLUX: IMPAIRED MUCOSAL INTEGRITY OR DISTINCT SENSORY INNERVATION?}

P Woodland* , C Lee, R Aktar, E Mthunzi, LA Blackshaw, SL Preston, D Sifrim. Barts and the London School of Medicine and Dentistry, Queen Mary University of London, London, UK

\subsection{6/gutjnl-2014-307263.67}

Introduction In patients with GORD, including refractory disease, reflux events reaching the proximal oesophagus are more likely to be perceived than those only reaching the distal oesophagus. There is also experimental data suggesting an increased sensitivity of the proximal oesophagus relative to the distal. As such, the proximal oesophagus is likely to be highly significant in the pathogenesis of GORD symptoms. Reasons for this proximal oesophageal sensitivity are not clear, but may include reflux volume, impairment in mucosal integrity or changes in sensory innervation. It has recently been shown that distal mucosal integrity (its ability to perform a protective barrier function) is more vulnerable to acid exposure in GORD than in controls. The integrity of the proximal oesophagus has not been tested. To our knowledge, there are no studies evaluating mucosal afferent innervation of the distal and proximal oesophagus. We aimed to compare mucosal integrity and afferent nerve distribution in the proximal and distal oesophagus in patients with heartburn without oesophagitis.

Methods In 23 patients with heartburn and 10 healthy volunteers baseline proximal and distal oesophageal impedance was measured in vivo. Oesophageal mucosal biopsies from the distal and proximal oesophagus were taken and baseline transepithelial electrical resistance (TER) was measured in Ussing chambers. Biopsies were examined immunohistochemically for presence and location of calcitonin gene-related peptide (CGRP) immunoreactive nerve fibres.

Results Baseline impedance was higher in the proximal than in the distal oesophagus in healthy volunteers $(2935 \pm 204 \Omega$ vs. $2234 \pm 290 \Omega, \mathrm{p}<0.05)$ and in patients $(2949 \pm 183 \Omega$ vs.1945 $\pm 235 \Omega, p<0.001)$. However, baseline TER was not significantly different between proximal and distal oesophagus, or between patients with heartburn and healthy volunteers. Mucosal CGRP-immunoreactive nerves were located more superficially in the proximal oesophagus compared to the distal oesophagus in healthy controls $(12.3 \pm 0.9$ vs. 23.8 \pm 1.2 cells from lumen, $\mathrm{p}<0.001)$ and in patients $(5.7 \pm$ 0.7 vs. $22.2 \pm 2.7$ cells from lumen, $\mathrm{p}<0.0001)$. Moreover, these nerves were located closer to the lumen in patients with heartburn compared to asymptomatic controls $(5.7 \pm 0.7$ vs. $12.3 \pm 0.9, \mathrm{p}<0.001)$.

Conclusion The baseline mucosal integrity of the proximal oesophagus is not more impaired than that of the distal, nor is it more impaired in patients with heartburn symptoms versus healthy controls.

Increased sensitivity of the proximal oesophagus in GORD may instead be associated with a more superficial location of mucosal afferent nerves. Topical protection of the proximal oesophageal mucosa is a potential treatment strategy to reduce this sensitivity.

Disclosure of Interest None Declared.

\section{OC-068 THE IMPACT OF ENDOSCOPIC THERAPY ON PATIENT-PERCEIVED OUTCOME AND QUALITY OF LIFE IN SPHINCTER OF ODDI DYSFUNCTION}

B Paranandi*, VTF Cheung, D Joshi, GH El-Sayed, GJ Johnson, SP Pereira, GJ Webster, MH Chapman. Pancreaticobiliary Medicine, University College London Hospitals, London, UK

\subsection{6/gutjnl-2014-307263.68}

Introduction Biliary Sphincter of Oddi dysfunction (SOD) is a benign but often debilitating condition. Significant improvement in pain following endoscopic sphincterotomy or sphincteroplasty (ES) in patients with Type 1 SOD, is excellent. Symptomatic improvement in patients with type 2 or 3 SOD is less favourable (reported $50-70 \%$ and $30-50 \%$ respectively). We aim to determine the impact of ES, on pain symptoms and global quality of life (QOL) in these groups, which has not previously been well defined.

Methods An ERCP database and electronic clinic lists (from September 2011 to 2013) were analysed to identify all cases of suspected SOD. Patients underwent a telephone questionnaire. The Glasgow Benefit Inventory (GBI), which assesses multiple physical, emotional and social parameters, was used to quantify global post-interventional QOL benefit. Total GBI scores can range from -100 (maximal negative benefit) to +100 (maximal positive benefit).

Results 163 new patients with suspected biliary SOD were identified of whom 89 underwent ERCP. 3 patients were excluded due to an alternative diagnosis at ERCP. The remaining cohort was predominantly Female (87\%) and

\begin{tabular}{llll} 
Abstract OC-068 Table 1 & \multicolumn{3}{c}{ Median GBI Scores (Post- ES) } \\
\hline Response to ESF & \multicolumn{3}{c}{ SOD subtype } \\
\hline & SOD1 & SOD2 & SOD3 \\
No improvement & -19.4 & -31 & -63 \\
Initial response then relapse & 8.3 & 8.3 & -19 \\
Sustained response & 44 & 31 & 29 \\
\hline
\end{tabular}

